\title{
Neue alte Herausforderungen
}

\author{
New and Old Challenges
}

Autoren

Institute
Thomas Becker ${ }^{1}$, Steffi G. Riedel-Heller ${ }^{2}$

Klinik für Psychiatrie und Psychotherapie II, Bezirkskrankenhaus Günzburg, Universität Ulm

${ }_{2}^{2}$ Institut für Sozialmedizin, Arbeitsmedizin und Public Health (ISAP), Medizinische Fakultät, Universität Leipzig

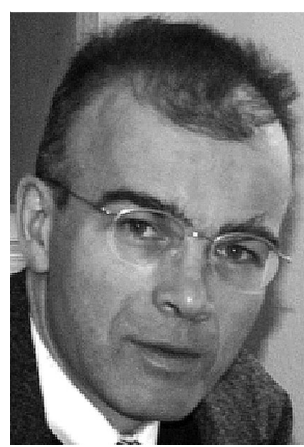

Prof. Dr. Thomas Becker

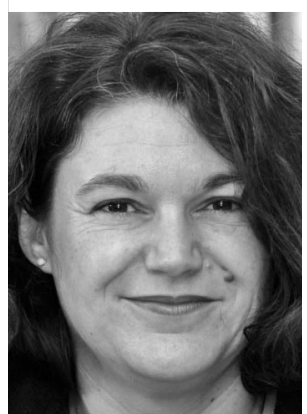

Prof. Dr. Steffi RiedelHeller

\section{Bibliografie}

DOI http://dx.doi.org/ 10.1055/s-0032-1327185 Psychiat Prax 2012; 39: 365-366 (c) Georg Thieme Verlag KG Stuttgart · New York ISSN 0303-4259

\section{Korrespondenzadresse}

Prof. Dr. med. Thomas Becker Ärztlicher Direktor, Klinik für Psychiatrie und Psychotherapie II der Universität Ulm, Bezirkskrankenhaus Günzburg Ludwig-Heilmeyer-Straße 2 89312 Günzburg t.becker@uni-ulm.de
Therapie und Praxis in Psychiatrie und Psychotherapie sollen mehrdimensional sein. Dies gilt generell für alle Formen und Verläufe psychischer Störungen, ohne Einschränkung auch für Menschen mit schweren psychischen Erkrankungen. Neben Pharmakotherapie und Psychotherapie stehen im therapeutischen "Werkzeugschrank“ des Faches Psychiatrie und Psychotherapie die psychosozialen Therapieverfahren. Diese sind schwer auf „einen Nenner“ zu bringen, sie imponieren durch Heterogenität. Die Deutsche Gesellschaft für Psychiatrie Psychotherapie und Nervenheilkunde (DGPPN) hat in ihrer Serie von DGPPN Behandlungsleitlinien neben den störungsbezogenen, diagnosespezifischen Leitlinien (aktuell die S3-Behandlungsleitlinie Bipolare Störungen [1]) auch eine S3-Leitlinie Psychosoziale Therapien bei Menschen mit schweren psychischen Störungen auf den Weg gebracht [2, 3]. Die Entscheidung gegen den Diagnose- oder Störungsbezug in diesem Leitlinienprojekt ist in Evidenz und Praxis begründet (und gegründet), denn tatsächlich bezieht sich ein großer Teil der Forschung zu den psychosozialen Interventionen auf die Zielgruppe der Menschen, die wiederholt und/oder länger und mit relevanten funktionalen (Alltags-)Konsequenzen erkranken [4]. Der diagnostisch wenig präzise bestimmten Zielgruppe der Menschen mit "severe mental illness“ ist somit eine pragmatische und „ökologische“ Validität nicht abzusprechen.

Das Projekt der Psychosozialen Therapie-Leitlinie ringt zum zweiten allerdings auch mit der Heterogenität der behandelten Themen, Perspektiven und Interventionen. Das Spektrum der Teilkapitel der Leitlinie umfasst zum einen Module oder Paradigmen therapeutischer Praxis wie "Milieutherapie“, „Empowerment“ und das „Recovery“-Konzept; es reicht über multidisziplinäre teambezogene „System“-Interventionen wie Case Management, Community Mental Health Teams, Assertive Community Treatment sowie (Crisis Interven- tion und) Home Treatment Teams bis hin zu den traditionellen psychosozialen Interventionen wie Psychoedukation (für Patienten und Angehörige), Bewegungs-, Sport- und Ergotherapie; von den Interventionen der Arbeitsintegration wie Supported Employment oder berufliche Rehabilitation bis hin zum Training sozialer Fertigkeiten sowie der Vielfalt künstlerischer Therapieangebote (Musik- und Kunsttherapie).

Zeitlich parallel hat der Gemeinsame Bundesausschuss (G-BA), der darüber entscheidet, welche Leistungen die gesetzliche Krankenversicherung (GKV) in Deutschland finanziert, das AQUA-Institut in Göttingen mit der Entwicklung eines Qualitätssicherungsverfahrens zur Versorgung bei psychischen Erkrankungen beauftragt. Der Schwerpunkt wird auf „schweren und schwersten Verläufen“ liegen. Soweit machbar, sollen Routinedaten in die Qualitätsmessung einfließen (www. aqua-institut.de; www.sqg.de). Dieses Projekt ist Teil einer breiter angelegten Politik im deutschen Gesundheitswesen zur Sicherung der Qualität der medizinischen Versorgung mittels objektivierbarer Merkmale. Weitere in diesem Kontext vom G-BA beauftragte sektorenübergreifende Qualitätssicherungsverfahren umfassen u.a. die (Katheter-)Koronarinterventionen, die Konisation in der Gynäkologie oder die Kataraktchirurgie in der Augenheilkunde.

Die Deutsche Gesellschaft für Psychiatrie, Psychotherapie und Nervenheilkunde (DGPPN) hat die Bedeutung des Themas erkannt und ein Projekt zur Entwicklung von Qualitätsindikatoren in Psychiatrie und Psychotherapie initiiert [5]. Qualitätsindikatoren für Projekte Integrierter Versorgung für Menschen mit schizophrenen Störungen wurden ebenfalls vorgelegt [6]. Die Akteure im psychiatrisch-psychotherapeutischen und psychosozialen Handlungs- und Forschungsfeld werden sich der Qualitätsherausforderung stellen müssen [7]. 
Welche Herausforderungen können sich aus diesen parallelen Entwicklungen ergeben?

1. Die Heterogenität psychosozialer Interventionen kann anregen, nach übergreifenden Linien oder Konstanten psychosozialer Therapeutik zu suchen. Wodurch etwa würde sich „individualisierte“ oder „targeted“ psychosoziale Therapie auszeichnen? Sind Grundlinien (a) über Dimensionen wie Selbstwirksamkeit oder „Recovery“-Orientierung, oder (b) über die „psychosoziale therapeutische Beziehung“, oder (c) über die Bedeutung von „Arbeit“ und „Arbeitsintegration“ zu identifizieren? Können sich diese Grundlinien mit den tragenden Pfeilern individualisierter oder störungsspezifischer Pharmako- und Psychotherapie zu einer Gesamttheorie von Therapie zusammenfügen?

2. Gibt es Qualitätsindikatoren, welche psychosozialen Interventionen besonders gerecht werden? Sollten wir Qualitätsindikatoren auf der „System“-Ebene fordern, welche z. B. Teamorientiertheit oder Multiprofessionalität oder Alltagsorientiertheit oder Inklusionsorientierung in komplexen Interventionen „messen“? Ist es wichtig, dass solche Qualitätsüberlegungen störungsspezifische Qualitätsindikatoren ergänzen?

3. Was müssen wir tun, um den Implementierungsgrad psychosozialer Interventionen zu bestimmen? Es ist Konsens, dass Leitlinien-Implementierung nicht als „Selbstläufer“ zu verbesserten Outcomes führt, dass sich enorme Implementierungslücken in der psychiatrisch-psychotherapeutischen und psychosozialen Versorgung auftun und dass die Evidenz-PraxisLücke ein ernstes Problem ist [8].

4. Können kleinere oder größere Projekte, welche die aktuelle Versorgungspraxis mit den Empfehlungen der neuen Psychosozialen S3-Leitlinie kontrastieren, bestehende Evidenz-Praxis-Lücken genauer benennen und die Praxis in ihrer Forderung nach Implementierung unterstützen? Könnte eine breiter angelegte Diskussion und Disseminierung der DGPPN S3Leitlinie Psychosoziale Therapien in der Breite der psychosozialen Träger und Diskussionsforen zusätzlichen Druck entfalten, damit die geschilderten Interventions- und Angebotsformen möglichst breit zum Einsatz kommen und den betroffenen Menschen zur Verfügung stehen?

5. Die Versorgungsforschung sollte sich der Frage stellen, ob in der Qualitätsdebatte und bei der Identifizierung zukunftsweisender Qualitätsindikatoren „systemische“ Aspekte abgebildet werden sollten, ob etwa der Grad der Integration im Versorgungssystem abgebildet oder gemessen werden sollte, um Qualität zu benennen oder zu verdeutlichen.

Aktuell weisen Qualitätsindikatorensätze, welche einerseits Basisindikatoren wie

- psychiatrische und somatische Komorbidität,
- jährliche kumulative Verweildauer,

- Regelungen zur Informationsübermittlung stationär/ambulant sowie

- Arbeit auf dem ersten Arbeitsmarkt [6], und

andererseits behandlungsbezogene Qualitätsindikatoren wie

- Anzahl der Suizide und Suizidversuche,

- rasche ambulante Terminvergabe nach Entlassung,

- Monitoring von Nebenwirkungen sowie

- Zwangsbehandlung [5]

umfassen, auf sektorübergreifende Aspekte in der Patientenversorgung hin.

Schließlich wissen wir, dass die Evidenz für Verbesserungen von Anbieter-Performance (Behandlungsprozess; process outcome) einerseits und Patientenbefinden (patient outcome) andererseits durch Leitlinien-Implementierung vorhanden, aber nicht überwältigend ist [8]. In den Arbeitsfeldern der Versorgungspraxis und -forschung gibt es unbestritten eine Spannung zwischen dem, was ist und dem, was sein sollte. Die Frage ist nun, ob die Verfügbarkeit der neuen DGPPN S3-Leitlinie „Psychosoziale Therapien“ und die anstehende Arbeit an Qualitätsindikatoren für die Versorgung von Menschen mit schweren psychischen Erkrankungen Anreiz bieten, um aus dieser Spannung Schwung für Innovation und Verbesserung zu schöpfen.

\section{Danksagung}

Dank an PD Dr. Anke Bramesfeld für die Durchsicht.

\section{Literatur}

1 Bauer $M$. Neue evidenz- und konsensbasierte S3-Leitlinie zur Diagnostik und Therapie bipolarer Störungen. Nervenarzt 2012; 83: 564-567

2 Deutsche Gesellschaft für Psychiatrie, Psychotherapie und Nervenheilkunde, Hrsg. S3 Praxisleitlinien in Psychiatrie und Psychotherapie. Leitlinie Psychosoziale Therapien bei Menschen mit schweren psychischen Erkrankungen. Heidelberg: Springer Verlag GmbH; im Druck

3 Pfennig A, Hölter G. Evidence-based medicine is gold standard for medical guidelines. Psychiat Prax 2011; 38: 218 - 220

4 Ruggeri $M$, Leese $M$, Thornicroft $G$ et al. Definition and prevalence of severe and persistent mental illness. Br J Psychiatry 2000; 177: 149-155

5 Zielasek J, Großimlinghaus I, Janssen B et al. Die Rolle von Qualitätsindikatoren in der psychiatrischen Qualitätssicherung und aktueller Stand der Entwicklung von Qualitätsindikatoren. Psychiatrie 2012; 9: 46 - 52

6 Weinmann S, Becker T. Qualitätsindikatoren für die Integrierte Versorgung von Menschen mit Schizophrenie. Handbuch. Gefördert vom AOK-Bundesverband. Bonn: Psychiatrie-Verlag; 2009

7 Steinert T, Eisele F, Längle G et al. PGI-I (patient's global impression) as an outcome and quality indicator of psychiatric in-patient treatment: results and concordance with doctor's assessments. Psychiat Prax 2010; 37: $343-349$

8 Weinmann S, Kösters M, Becker T. Effects of implementation of psychiatric guidelines on provider performance and patient outcome: systematic review. Acta Psychiatr Scand 2007; 115: 420-433 